\title{
LA INFLUENCIA DEL FERROCARRIL EN EL DESARROLLO URBANO ESPAÑOL (1860-1910)*
}

\author{
RAFAEL BARQUÍN \\ UNED \\ PEDRO PÉREZ \\ UNED \\ BASILIO SANZ \\ $\mathrm{UNED}^{\mathrm{a}}$
}

Railways' influence in the Spanish urban development

\begin{abstract}
The aim of this paper is to measure the influence of the railroad in the urbanization of Spain between 1860 and 1910. Our sources are from quantitative information -censuses of population- and qualitative one -coastal condition, existence of mining industry or industry, administrative capital and date of the railway connection-. We have estimated a first model of data panel in differences. Based on this model we have employed different estimation techniques in order to address omitted variables and/or endogeneity of the train variable. Results from all estimations give us clear evidence of the positive influence of the railroad on the urban growth. In addition a quasiexperiment design reinforces this conclusion. In short, although moderate, our paper shows strong evidence of the influence of the railroad on Spanish urbanization. This conclusion is coherent with other research.
\end{abstract}

Keywords: urbanization, railways, panel data

JEL Codes: C53, J11, N73, R15

* Received 26 February 2012. Accepted 31 October 2012. Los autores agradecen a Mariano Matilla García la colaboración prestada, así como los comentarios y las observaciones de los dos evaluadores anónimos de la RHE-JILAEH.

a Universidad Nacional de Educación a Distancia. Departamentos de Economía Aplicada e Historia Económica y de Economía Aplicada Cuantitativa I. Facultad de Ciencias Económicas y Empresariales, c/ Senda del Rey, 11, 28040, Madrid. Correos electrónicos: rbarquin@cee.uned.es, pperez@cee.uned.es y bsanz@cee.uned.es. 


\section{RESUMEN}

El propósito de este artículo es medir la influencia del ferrocarril en el crecimiento urbano en España entre 1860 y 1910. A partir de información cuantitativa - censos de población- y cualitativa - condición costera, existencia de minería o industria, capitalidad administrativa y fecha de la conexión ferroviaria-, se construye un primer modelo de diferencias en datos de panel. Sobre la base de este modelo se utilizan distintas técnicas de estimación para tratar de solventar la existencia de variables omitidas y la posible endogeneidad de la variable «tren». Los resultados de todas las estimaciones muestran una evidencia clara de la influencia positiva del ferrocarril sobre el crecimiento urbano. Esta conclusión resulta además reforzada con la estimación de un segundo modelo basado en el diseño de un cuasi experimento. En resumen, aunque moderada, la influencia del ferrocarril parece sólidamente establecida desde el punto de vista empírico. Este resultado es coherente con otras investigaciones.

Palabras clave: urbanización, ferrocarril, datos de panel

\section{INTRODUCCIÓN}

El propósito de este artículo es medir la influencia de la construcción de la red ferroviaria en el desarrollo urbano de España durante la segunda mitad del siglo XIX. Para ello es necesario resolver dos problemas relacionados entre sí. En primer lugar, hay que especificar correctamente el modelo que relaciona esas variables. La urbanización fue propiciada por un amplio conjunto de factores de los que la red ferroviaria sólo fue uno. Por tanto, existe el riesgo de que un modelo incompleto sobrevalore sus efectos al omitir variables significativas. El segundo problema radica en que la llegada del ferrocarril no sólo incidió en la urbanización, sino que también ésta influyó en el diseño de la red; es decir, el ferrocarril podría ser tanto una variable exógena como endógena. Plantearemos varios modelos que tratarán de comprobar y resolver estos problemas. Antes haremos un repaso de la bibliografía disponible (apartado 2), examinaremos sumariamente el proceso de urbanización y construcción de la red ferroviaria (apartado 3), y analizaremos los datos que podemos emplear (apartado 4).

En la España del siglo XIX existía la convicción de que la construcción de una red ferroviaria no sólo era imprescindible para el desarrollo económico (y, por ende, urbano), sino que tendría efectos poco menos que inmediatos. De ahí que hubiera intensas polémicas sobre el trazado de las líneas, así como una fuerte implicación de los poderes locales. Lo cierto es que desde entonces se han ofrecido explicaciones teóricas muy sólidas para justificar 
esa relación causal. Ésta se puede fundamentar, por ejemplo, a través de la teoría schumpeteriana del ciclo económico, que considera el desarrollo y la progresiva implantación del ferrocarril como uno de los factores determinantes de los grandes ciclos económicos ${ }^{1}$. También se puede contemplar el fenómeno desde la integración de los mercados. La mejora del abastecimiento de las ciudades fue un elemento decisivo en el crecimiento urbano; primero como instrumento para la superación de las crisis de mortalidad y, más adelante, para la formación de grandes urbes ${ }^{2}$.

Desde otro enfoque, la mejora de los transportes habría generado un mayor crecimiento urbano por sus eslabonamientos (linkages) con otras actividades económicas: hacia atrás por la expansión de la siderurgia y la minera del carbón, y hacia delante por la apertura de nuevos mercados a la producción fabril ${ }^{3}$. Esto último nos conduce al ahorro social. La sustitución de un medio de transporte tradicional y caro por otro moderno y más económico supone una mejora que, en el contexto del siglo XIX europeo, debería haber provocado un mayor crecimiento industrial y urbano. Por tanto, la medición de los efectos del ferrocarril sobre la urbanización debería servir para verificar la validez de algunas estimaciones del ahorro social; lo que resulta muy pertinente en el caso español ya que las realizadas hasta ahora presentan diferencias considerables ${ }^{4}$.

Así pues, la conexión entre ferrocarril y crecimiento urbano no sólo constituyó un asunto político importante en la Europa (y en la España) del siglo XIX; en sí mismo es un asunto relevante para la investigación en Historia Económica. Pero, como veremos, esa relación es tan aparentemente obvia como difícil de probar de un modo riguroso.

\section{ESTADO DE LA CUESTIÓN}

Hace más de un siglo, Adna Ferrin Weber (1899, pp. 169-228), apoyándose en la base estadística disponible para los principales países europeos y Estados Unidos, identificó los factores que explicarían la progresiva concentración de la población a lo largo del siglo XIX. Éstos serían la centralización administrativa y comercial, la industrialización y, de modo indirecto, el ferrocarril. Los mismos factores han sido reiterados en la bibliografía posterior, aunque la importancia que se ha dado a cada uno de ellos ha variado. Por ejemplo, para la Italia de l'Unità, Lorenzo del Panta (1989, pp. 263-264) daba preferencia a la centralización administrativa sobre el crecimiento industrial y otorgaba al ferrocarril un papel modesto. Para Jean Pierre Poussou (1989, pp. 77-80), la urbanización en Francia fue propiciada por la centralización política,

\footnotetext{
1 Véase Schumpeter (1939, pp. 215 y ss.).

2 Véase Bairoch (1988, pp. 278 y ss.).

3 Véase Hirschman (1958).

4 Véanse Herranz (2012), Barquín (1999) y Gómez Mendoza (1982).
} 
administrativa y comercial, y la reducción de los costes de transporte - es decir, la construcción del ferrocarril-; pero también por el desarrollo del comercio marítimo y la presencia de actividades económicas específicas pero no necesariamente industriales, como el turismo. Sin embargo, Denise Pumain (1982, pp. 547-548) no creía que se pudiera confirmar la influencia del ferrocarril en la urbanización francesa. Para el conjunto de Europa, Bairoch y Goertz (1986) alcanzaban una conclusión similar. En la estimación de su modelo la variable «ferrocarril» no era significativa, y por tanto debía ser rechazada como explicativa de la urbanización ${ }^{5}$. De todos modos, muchos de esos estudios ofrecen resultados poco sólidos porque carecen de una metodología econométrica adecuada.

Pero, en los últimos años, este campo de investigación ha experimentado cambios notables. La Nueva Geografía Económica (NEG), cuyo arranque podemos situar en el trabajo de Krugman (1991), supuso una renovación tanto de los temas de investigación (por ejemplo, los relativos a la localización) como de su metodología. Las nuevas investigaciones se han visto auxiliadas por el reciente desarrollo de amplias bases de datos con información geográfica, lo que ha dado lugar al llamado HGIS (Historical Geographic Information System). La combinación de nuevos enfoques y técnicas con esas bases de datos ha generado valiosas aportaciones. Trabajos como los de Gregory y Martí-Henneberg (2010) para Inglaterra y Gales entre 1825 y 1911, Kotavaara et al. (2011) para Finlandia entre 1880 y 1970, o Akgüngör et al. (2011) para Turquía entre 1856 y 2000, ponen de manifiesto que el acceso a la red ferroviaria no fue indiferente a los procesos de concentración demográfica. Por ejemplo, Gregory y Martí-Henneberg (2010) señalan que, en conjunto, las poblaciones con ferrocarril crecieron un 3,6\% más por década durante el periodo 1841-1911. La mayor debilidad de esos estudios es que la relación de causalidad no aparece satisfactoriamente resuelta.

Desde una perspectiva más econométrica, algunos trabajos recientes resultan especialmente atractivos para los fines perseguidos en este artículo. Así, Atack et al. (2010) han estudiado el impacto de la construcción de la red ferroviaria sobre la población urbana en Estados Unidos entre 1850 y 1870. Hornung (2012), el de los ferrocarriles prusianos en el crecimiento urbano entre 1838 y 1871. En los dos casos se reconoce un efecto positivo y significativo del acceso a la red ferroviaria. Por ejemplo, Hornung (2012) concluye que las ciudades conectadas al ferrocarril en 1848 incrementaron el porcentaje de su crecimiento anual en un $0,9 \%$ durante las siguientes dos décadas ${ }^{6}$.

5 Lo que no parecía sensato a los propios autores. En un trabajo posterior, Bairoch (1988, pp. 262-264 y 276-277) rechaza esta conclusión.

6 Otro trabajo interesante, por el empleo de esta metodología, es el de Banerjee, Duflo y Qian (2012) sobre los ferrocarriles en China entre 1986 y 2006. En este caso, la variable explicada no es la urbanización, sino el PIB per cápita. 
Esos trabajos no contemplan, expresamente, los efectos que sobre el crecimiento urbano tuvieron otros factores. En este sentido, resulta interesante la investigación de Huijg, Koopmans y Rietveld (2010) sobre la construcción de la red ferroviaria en los Países Bajos entre 1840 y 1930, que pone el acento en la medición de la accesibilidad a los centros urbanos. Los autores concluyen que la construcción del ferrocarril habría tenido efectos positivos pero muy modestos en el crecimiento urbano, inferiores a los derivados de la propia densidad urbana (en sentido negativo) y la proximidad a las grandes ciudades (en sentido positivo) ${ }^{7}$. De todos modos, la singularidad de los Países Bajos, una nación densamente poblada y muy urbanizada desde la Baja Edad Media, hace difícil trasladar esa metodología a España y, en realidad, a la mayor parte de Europa.

Las investigaciones realizadas en España han llevado cierto retraso con respecto a sus correspondientes de Europa y Estados Unidos. Primero se hizo un trabajo de identificación de las variables que podrían explicar el crecimiento urbano. De ello se extrajo una lectura que sólo más tarde, e indirectamente, fue retada. El crecimiento urbano habría tenido lugar en un país con un pobrísimo desarrollo industrial y, por tanto, sin una aportación significativa del grupo socioprofesional que más contribuyó al crecimiento urbano, el de los obreros industriales. Así, Gómez Mendoza y Luna Rodrigo (1986, p. 18) no creen posible establecer un vínculo entre urbanización y desarrollo industrial en la mayor parte de los casos. Pérez Moreda (1985, pp. 53-55) explica la urbanización en el periodo 1836-1930 por los cambios en la estructura administrativa (la capitalidad de provincias) y, sólo a escala local o regional, por la industrialización y la explotación minera. Rodríguez Osuna (1985, pp. 125-164), para el siglo XX más que para el XIX, sí que otorga cierto papel a la industrialización temprana; pero presta más atención a otros factores, como la dicotomía costa-interior y la capitalidad provincial. Todos conceden poca importancia a las mejoras en la red ferroviaria.

Sin embargo, esta postura ha sido criticada en los últimos tiempos desde los nuevos enfoques historiográficos de la NEG. Así, Mojica y Martí-Henneberg (2011) han estudiado la influencia del ferrocarril sobre la urbanización de Francia, España y Portugal mediante una amplia base HGIS alcanzando conclusiones generalizables a los tres países. En su opinión, el ferrocarril contribuyó poderosamente a la concentración de la población tanto en un sentido positivo — crecimiento urbano- como negativo — despoblamiento de las áreas no conectadas-. Más recientemente, Morillas-Torné et al. (2012), y con la misma metodología, han alcanzado conclusiones semejantes aplicables específicamente al caso español. La principal aportación con respecto al trabajo anterior es que la intensidad de ese proceso se encontraría estrechamente relacionada con el tamaño de los núcleos urbanos.

7 Estas conclusiones son consistentes con las realizadas en trabajos anteriores, como las de de Rietveld y Bruinsma (1998). 
Por otro lado, han aparecido varios trabajos que emplean técnicas econométricas sofisticadas, de los que cabe extraer varias conclusiones sobre el problema que aquí se plantea, aunque no lo aborden directamente. Por ejemplo, Herranz Loncán (2009) no encuentra efectos significativos de la inversión en infraestructuras de transporte en la productividad laboral o la inversión en maquinaria. Independientemente de la explicación que pueda darse (el autor propone dos) lo relevante es que este resultado no invita a esperar un fuerte crecimiento urbano de la construcción de la red ferroviaria.

Asimismo, los trabajos de Barquín (1997) y Matilla, Pérez y Sanz (2009) describen un proceso más o menos continuo de integración de mercados durante la segunda mitad del siglo XIX, atribuible en gran parte a las mejoras en los transportes. Sería razonable esperar que este proceso favoreciera la especialización regional y la industrialización de algunas regiones, así como una creciente desigualdad en la renta ${ }^{8}$. Y, en fin, que favoreciese procesos de urbanización. Por ejemplo, Silvestre (2005) sostiene que el coste del transporte fue una variable muy significativa para explicar por qué los movimientos migratorios internos entre 1860 y 1930 no fueron mayores. El correlato obvio es que sin la construcción de una red ferroviaria nacional el crecimiento urbano hubiera sido aún menor. De modo semejante, las investigaciones realizadas sobre la localización y concentración industrial revelan la importancia de factores estrechamente relacionados con la construcción de la red ferroviaria. Así, para el periodo 1856-1929 Martínez-Galarraga (2012) señala que el mercado potencial de cada región, creciente con la mejora de los transportes y la integración del mercado, fue un factor relevante en la localización industrial. Pons et al. (2007 y 2009) prueban la existencia de una relación directa entre la localización de los lugares a los que los trabajadores emigraban y el mercado potencial de esas regiones.

En resumen, existe un gran número de investigaciones que sugieren la existencia de una relación directa entre construcción ferroviaria y crecimiento urbano en España ${ }^{9}$. No obstante, de las mismas no es posible extraer conclusiones claras sobre la intensidad del fenómeno.

\section{EL DESARROLLO DE LA RED URBANA Y FERROVIARIA DE ESPAÑA EN LA SEGUNDA MITAD DEL SIGLO XIX}

La primera dificultad a la que se enfrenta un trabajo de esta naturaleza es determinar qué era una ciudad en la España de la segunda mitad del siglo XIX. En los estudios comparados sobre la urbanización en Europa suele

\footnotetext{
${ }^{8}$ Véanse Tirado, Pons y Paluzie (2006) y Rosés, Martínez-Galarraga y Tirado (2010).

${ }^{9}$ En realidad, la hipótesis de una relación causal entre desarrollo ferroviario y urbano es comúnmente asumida desde enfoques muy alejados de la cliometría. Por ejemplo, Capel (2011) analiza con detenimiento cómo la propia morfología de las ciudades se vio alterada por la llegada del ferrocarril.
} 


\section{CUADRO 1 \\ PARTICIPACIÓN DE LA POBLACIÓN URBANA EN ESPAÑA SEGÚN VARIOS CRITERIOS}

\begin{tabular}{|l|c|c|c|c|c|}
\hline & $\mathbf{1 8 6 0}$ & $\mathbf{1 8 7 7}$ & $\mathbf{1 8 8 7}$ & $\mathbf{1 9 0 0}$ & $\mathbf{1 9 1 0}$ \\
\hline $\mathrm{CU}>5.000$ & & & 33,0 & 35,5 & 37,4 \\
\hline $\mathrm{TM}>8.000 \mathrm{CU}>5.000$ & & & 28,9 & 31,3 & 33,8 \\
\hline $\mathrm{TM}>10.000 \mathrm{CU}>5.000$ & & & 25,8 & 29,0 & 31,0 \\
\hline $\mathrm{TM}>10.000+$ cap. & 21,0 & 24,4 & 25,8 & 28,8 & 30,5 \\
\hline $\mathrm{TM}>20.000+$ cap. & 15,0 & 17,5 & 18,6 & 22,6 & 23,5 \\
\hline
\end{tabular}

Fuente: elaboración propia a partir de Luna (1988) y censos del INE.

Nota: CU, casco urbano; TM, término municipal; cap., capitalidad.

establecerse un criterio de demarcación basado únicamente en el tamaño. El límite de los 5.000 habitantes ha sido corriente, si bien algunos autores han empleado otros más bajos. Claro que en Estados Unidos es normal que ese umbral se sitúe en los 8.000 habitantes. Por supuesto, todo criterio es discutible, y su aplicación tiene más o menos sentido atendiendo a la época histórica o al propósito del estudio.

En cualquier caso, es muy llamativo que en España se establezcan criterios más rigurosos que en el resto de Europa. Así, ese límite superior de 5.000 habitantes, habitual en Europa, viene a ser el límite inferior en España, donde varios especialistas han propuesto umbrales de $10.000 \mathrm{y}$ 20.000 habitantes, a veces auxiliados por otros criterios ${ }^{10}$. Se argumenta que muchas aglomeraciones del arco mediterráneo eran lo que David Reher denominaba «agrociudades»; es decir, núcleos de población en los que la mayor parte de sus habitantes tenían su principal actividad económica en el campo circundante, por lo que no eran «verdaderas» ciudades. Por otro lado, en el norte y noroeste peninsular existían municipios amplios y densamente poblados cuyos habitantes vivían de la agricultura y la ganadería en pequeños núcleos dispersos. En realidad, la consideración de una aglomeración como urbana exigiría atender a cuestiones relativas a los servicios públicos y el acceso a bienes y servicios que determinarían diferentes umbrales en cada época.

Obviamente, el empleo de uno u otro criterio condiciona mucho el peso de la población urbana sobre el conjunto del país. El Cuadro 1 recoge esa participación según varios censos y criterios, algunos de los cuales han sido empleados por otros autores. El recorrido de los datos es considerable. Por ejemplo, y según los datos del censo de 1887, la población urbana en España

${ }^{10}$ Véanse Del Campo (1972), Rodríguez Osuna (1985, pp. 26-28) y Reher (1986). 
CUADRO 2

PARTICIPACIÓN DE LA POBLACIÓN URBANA SOBRE EL TOTAL NACIONAL

\begin{tabular}{|l|r|r|r|c|c|}
\hline & $\mathbf{1 8 5 0}$ & $\mathbf{1 8 7 0}$ & $\mathbf{1 9 0 0}$ & $\mathbf{1 9 1 3}$ & $\mathbf{1 9 1 3 / 1 8 5 0}$ \\
\hline Alemania & 15,0 & 24,5 & 42,0 & 51,0 & 3,4 \\
\hline Austria-Hungría & 9,7 & 12,1 & 25,6 & 29,4 & 3,0 \\
\hline Balcanes* & 10,5 & 11,8 & 19,8 & 22,6 & 2,2 \\
\hline Bélgica & 33,5 & 38,8 & 52,3 & 58,0 & 1,7 \\
\hline España & 18,0 & 22,5 & 34,0 & 39,3 & 2,2 \\
\hline Francia & 19,5 & 24,3 & 35,4 & 39,5 & 2,0 \\
\hline Italia & 23,0 & 25,0 & 35,5 & 41,5 & 1,8 \\
\hline Países Bajos & 35,6 & 38,5 & 47,8 & 51,3 & 1,4 \\
\hline Portugal & 15,0 & 15,0 & 15,7 & 15,6 & 1,0 \\
\hline Reino Unido & 39,6 & 53,3 & 67,4 & 69,7 & 1,8 \\
\hline Rusia & 7,2 & 9,2 & 13,2 & 14,6 & 2,0 \\
\hline Suecia & 6,8 & 9,9 & 19,3 & 23,7 & 3,5 \\
\hline Suiza & 11,9 & 17,5 & 30,6 & 39,3 & 3,3 \\
\hline Europa (sin Rusia) & 16,4 & 20,8 & 33,2 & 38,7 & 2,4 \\
\hline Europa occidental & 24,1 & 32,8 & 47,6 & 53,4 & 2,2 \\
\hline Europa medit. & 20,5 & 23,2 & 33,1 & 38,3 & 1,9 \\
\hline
\end{tabular}

Fuente: Bardet y Dupâquier (1998, p. 200).

*Incluye a Bulgaria, Grecia, Rumania y Serbia.

habría oscilado entre el 18,6\% (localidades con más de 20.000 habitantes y capitales de provincia, independientemente de su tamaño) y el 33,0\% (localidades con un casco urbano superior a 5.000 habitantes).

El Cuadro 2 recoge esa participación de la población urbana en varios países europeos. Ha sido elaborado por Bardet y Dupâquier (1998, pp. 200-205) apoyándose en gran medida en Bairoch (1988, pp. 219-221) y Bairoch y Goertz (1986). Hay que advertir que el propio Bairoch cree que en sus cifras hay un cierto grado de arbitrariedad por la elección de ciudades y que los datos relativos a España tienen un considerable margen de error. $\mathrm{Si}$, pese a todo, les otorgamos cierta credibilidad, se puede concluir que el peso de las ciudades en España en la segunda mitad del siglo XIX no fue pequeño. Y también que el crecimiento urbano en España fue semejante al de Europa occidental. En uno y otro ámbito, la participación de éstas sobre el total se multiplicó entre 1850 y 1913 por 2,2. Sin embargo, para un periodo un poco más corto (1860-1910) y según los datos del Cuadro 1, ese factor 
sería sensiblemente menor: alrededor de 1,5. Esa misma impresión de un proceso de urbanización relativamente modesto se desprende de la bibliografía española ${ }^{11}$. En fin, es posible que las comparaciones entre países hayan sobrevalorado el crecimiento urbano en España.

Sea como fuere, hubo un nítido crecimiento urbano; pero resulta difícil identificar sus causas. De la comparación de los censos de 1860 y 1900 sólo se pueden extraer dos conclusiones genéricas. Primera, que el crecimiento fue la norma, y el decrecimiento puntual y bastante impredecible. Alrededor del $85 \%$ de las ciudades españolas ganaron población. Y sólo menguó una de cierta importancia, Cádiz. Precisamente en el suroeste peninsular se ubican muchas localidades que perdieron habitantes; pero hay ciudades menguantes en casi cualquier punto de la geografía española. La segunda conclusión genérica es que no debió haber un único factor explicativo. Como es lógico, las mayores tasas de crecimiento corresponden a ciertas localidades mineras. Pero, inmediatamente detrás, aparecen otras cuya expansión podría explicarse, a priori, por su puerto, su condición de mercado comarcal, o su función como centro administrativo. En resumen, la urbanización española aparece como un fenómeno amplio, complejo y exitoso.

El crecimiento de la red ferroviaria española tuvo características diferentes. Antes de 1850, apenas había $29 \mathrm{~km}$ abiertos al tráfico ferroviario, los correspondientes a la línea Barcelona-Mataró. En los cinco años siguientes, se abrieron otros $400 \mathrm{~km}$. Pero no fue hasta la aprobación de la ley ferroviaria de 1855 cuando la construcción tomó impulso. En 1865, ya existían alrededor de cinco mil kilómetros, pero la crisis de aquel año y la guerra carlista paralizaron las obras. A partir de 1875, se dio un nuevo empuje a la construcción, aunque menos intenso que el anterior. Hacia 1910, España contaba con once mil kilómetros de ferrocarriles de vía ancha, y más de tres mil kilómetros de vía estrecha ${ }^{12}$. En comparación con Europa, el ritmo de construcción fue muy elevado durante el periodo 1860-1880: un 7\% acumulado anual. Entre los grandes países de Europa occidental, sólo Suecia tuvo una tasa de crecimiento más elevada. Todo esto explica la rápida reducción del déficit de infraestructuras en los primeros años. En 1860 la densidad de la red ferroviaria española era un $29,4 \%$ de la europea; en 1870 ya era del $45,3 \%$. Sin embargo, en las siguientes décadas ese porcentaje se mantuvo más o menos estable.

Así pues, la red ferroviaria española apareció más tarde que en la mayor parte de Europa, pero se desarrolló con bastante rapidez. España pudo dotarse de un ferrocarril modesto, en comparación con los del resto del resto de los países europeos, pero razonable para su nivel de riqueza y población. Incluso puede hablarse de cierta sobreinversión. Herranz (2008, pp. 51-53) ha probado que existe una correlación bastante estrecha entre el nivel de desarrollo económico y la extensión de la red ferroviaria. Para una muestra de 19/20 países existiría una

11 Por ejemplo, Valero Lobo (1989, pp. 14-16) o Gómez Mendoza y Luna (1986, p. 18).

12 Véase Herranz (2004, p. 35). 


\section{CUADRO 3}

DENSIDADES FERROVIARIAS EN EUROPA Y TASAS DE CRECIMIENTO

\begin{tabular}{|l|r|r|r|r|r|r|c|c|}
\hline & \multicolumn{6}{|c|}{ Densidad ferroviaria } & \multicolumn{2}{c|}{ Tasa de crecimiento } \\
\cline { 2 - 9 } & $\mathbf{1 8 6 0}$ & $\mathbf{1 8 7 0}$ & $\mathbf{1 8 8 0}$ & $\mathbf{1 8 9 0}$ & $\mathbf{1 9 0 0}$ & $\mathbf{1 9 1 0}$ & $\mathbf{1 8 6 0 / 1 8 8 0}$ & $\mathbf{1 8 8 0 / 1 9 1 0}$ \\
\hline Alemania & 21,0 & 35,7 & 62,6 & 79,3 & 95,6 & 113,2 & 5,6 & 3,0 \\
\hline Austria & 9,8 & 20,5 & 38,4 & 51,5 & 65,5 & 77,8 & 7,1 & 3,6 \\
\hline Bélgica & 58,7 & 98,4 & 139,6 & 177,6 & 216,7 & 283,3 & 4,4 & 3,6 \\
\hline España & 3,8 & 10,9 & 14,8 & 19,8 & 26,2 & 29,0 & 7,0 & 3,4 \\
\hline Francia & 17,8 & 33,4 & 48,2 & 69,4 & 81,0 & 93,5 & 5,1 & 3,4 \\
\hline Hungría & 5,0 & 10,7 & 21,8 & 34,6 & 52,6 & 63,4 & 7,7 & 5,5 \\
\hline Italia & 8,4 & 21,9 & 29,7 & 45,7 & 55,3 & 58,7 & 6,5 & 3,5 \\
\hline Países Bajos & 9,8 & 41,5 & 53,8 & 76,3 & 81,0 & 93,2 & 8,9 & 2,8 \\
\hline Portugal & 0,7 & 7,7 & 12,3 & 20,8 & 23,4 & 26,4 & 15,3 & 3,9 \\
\hline Reino Unido & 63,5 & 93,8 & 109,0 & 121,1 & 130,9 & 140,0 & 2,7 & 1,3 \\
\hline Rusia & 0,3 & 2,2 & 4,7 & 6,3 & 10,9 & 13,6 & 14,2 & 5,5 \\
\hline Suecia & 2,3 & 7,5 & 25,6 & 35,0 & 49,3 & 60,3 & 12,8 & 4,4 \\
\hline Suiza & 25,5 & 34,4 & 61,0 & 75,2 & 88,8 & 111,5 & 4,5 & 3,1 \\
\hline Europa & 12,9 & 24,0 & 35,1 & 47,1 & 57,8 & 67,0 & 5,1 & 3,3 \\
\hline España/Europa & 29,4 & 45,3 & 42,0 & 42,0 & 45,3 & 43,2 & & \\
\hline
\end{tabular}

Fuente: Herranz (2008, p. 49).

\section{CUADRO 4}

TASAS DE CRECIMIENTO DE LA RED FERROVIARIA ESPAÑOLA. VÍA ANCHA Y ESTRECHA

\begin{tabular}{|l|c|c|c|}
\hline $1860 / 1870$ & 11,2 & $1860 / 1877$ & 7,5 \\
\hline $1870 / 1880$ & 3,1 & & \\
\hline $1880 / 1890$ & 3,0 & $1877 / 1887$ & 3,9 \\
\hline $1890 / 1900$ & 2,7 & $1887 / 1900$ & 2,5 \\
\hline $1900 / 1910$ & 1,1 & & \\
\hline
\end{tabular}

Fuente: Anes (1978, pp. 485-486) y Herranz (2004, p. 35).

relación muy estable entre densidad ferroviaria (kilómetros de vía férrea por kilómetro cuadrado de superficie territorial) y densidad económica (PIB por kilómetro cuadrado). Así, el coeficiente de determinación que regresa esas dos 
variables es 0,96 y 0,93 en 1870 y 1900, respectivamente. Pues bien, en 1870, España disponía de una dotación de infraestructuras ferroviarias algo superior a lo que cabría esperar de su posición; y dentro de lo previsible en 1900. Se observa una mayor diferencia en la calidad del servicio, en franco descenso desde finales de siglo, tal y como sugiere, por ejemplo, la pobrísima dotación de vías dobles. En todo caso, España no se aparta significativamente de otros países de la Europa mediterránea.

\section{LA INFORMACIÓN EMPLEADA}

La medición de la influencia del ferrocarril en el crecimiento de la población urbana durante la segunda mitad del siglo XIX plantea dificultades relacionadas, sobre todo, con la falta de información desagregada. Por ese motivo, nos hemos visto obligados a recurrir a modelos econométricos de variables cualitativas que necesariamente muestran niveles explicativos más bajos que aquellos en los que intervienen variables cuantitativas ${ }^{13}$. En resumen, utilizaremos datos cuantitativos para la variable endógena - la poblacióny cualitativos en la exógena, el ferrocarril y las otras variables de control capitalidad, existencia de minería o industria y condición costera.

Como hemos visto, la variable «población» encierra problemas tanto de carácter documental como por la misma elección de un criterio razonable que defina lo que es una ciudad. Con respecto a lo primero, decidimos partir del censo de población de 1887 por su confiabilidad ${ }^{14}$. Además, se sitúa, aproximadamente, en el centro del periodo de estudio, cuando el ferrocarril ya había alcanzado la gran mayoría de las urbes grandes y medianas. Pero, sobre todo, es el primero en el que contamos con información detallada sobre los cascos urbanos ${ }^{15}$. Esto es importante porque el tamaño del casco urbano en España es, a priori, la condición fundamental para distinguir las verdaderas ciudades de los términos municipales dispersos del noroeste. No obstante, este criterio puede ser insuficiente para discriminar las agrociudades del Mediterráneo y del sur del país. De ahí que hayamos considerado conveniente incorporar un segundo criterio: la misma población del término municipal. La hipótesis sobre la que nos basamos es que el mero tamaño implica una mayor diversificación de actividades y, por tanto, supone un argumento a favor de su condición urbana.

Dado el propósito de este trabajo, hemos buscado unos umbrales que permitan construir una muestra de ciudades lo bastante grande como para ser representativa; pero que también aseguren la condición de urbanidad. En definitiva, «nuestra» ciudad se define como aquella aglomeración con más de 8.000 habitantes en su término municipal y más de 5.000 en su casco

\footnotetext{
13 En el sentido de modelos econométricos con coeficientes de determinación $R^{2}$ menores

14 Véase Reher, Nogueras y Pombo (1993).

15 Véase Luna (1988)
} 
urbano. Esto permite obtener una muestra de más de 200 casos con un grupo de control -localidades sin estación de tren-que nunca es inferior a $45^{16}$.

También se hicieron censos en los años 1857, 1860, 1877, 1900 y 1910. Excluidos los de 1857 y 1897 por su menor calidad y por estar muy próximos a otros mejores, disponemos de cinco cortes temporales con los que describir la evolución demográfica urbana en España. Desafortunadamente, la distancia que separa unos censos de otros no es uniforme: 17, 10, 10, 13 y 10 años. La muestra de ciudades de 1887 debe extenderse hacia atrás y hacia adelante, lo que plantea dos tipos de dificultades. En primer lugar, podríamos tomar como ciudades a municipios que no reunían un casco urbano de 8.000 habitantes en 1860 y 1877, pero sí en 1887. Además, como desconocemos el tamaño del casco urbano en esos dos censos, ni siquiera sabríamos si cumplen el segundo criterio. No hay forma de resolver este problema, si bien no parece grave. Por ejemplo, en Galicia y Asturias, donde estos problemas de identificación son serios, sólo identificamos diez ciudades: las cinco capitales y Vigo, Santiago, Ferrol, Avilés y Gijón. Parece bastante claro que todas ellas fueron ciudades plenas a lo largo de toda la segunda mitad del siglo XIX.

La segunda dificultad se deriva de la agrupación o segregación de algunas localidades. De nuevo, es un problema menor pues afecta a una parte muy pequeña de la muestra. El criterio general ha sido tomar el censo de 1887 como referente de todos ellos. Por ejemplo, si una localidad era independiente antes de ese año, pero aparece fusionada en 1887, la población considerada en 1860 y 1877 es la correspondiente a la suma de las dos localidades ${ }^{17}$. Éste es, con diferencia, el caso más frecuente. Excepcionalmente aparecen situaciones más complicadas; por ejemplo, cuando dos localidades se unen con posterioridad a ese año. En estos casos, la población de 1900 y 1910 pierde la parte proporcional de esa localidad de acuerdo al censo de 1887.

Como variable endógena del modelo hemos tomado la tasa de variación media intercensal, es decir, la tasa de variación porcentual de la población de cada municipio (i) en el periodo intercensal $(t)$, dividida por el número de años transcurridos entre los censos. De esta manera, los datos obtenidos son homogéneos. El empleo de porcentajes en lugar de niveles elimina, o al menos atenúa, cualquier posible problema de no-estacionariedad, además de

16 De esa muestra hemos detraído las ciudades canarias. El fuerte crecimiento urbano en las islas (entre 1860 y 1910, Las Palmas y Santa Cruz de Tenerife cuadruplicaron su población) obedeció a circunstancias muy particulares y distintas de las de la península, relacionadas con las migraciones y el comercio con América. Por otro lado, nunca contaron con un ferrocarril; pero, de haberlo tenido, tampoco habrían podido participar de la red nacional. Esto las situaría dentro del grupo de control, pero con características muy diferentes de otras ciudades del mismo grupo.

17 No obstante, el área metropolitana de Barcelona (Gracia, Sans, San Andrés y San Martín) ha sido considerada como un todo por el gran dinamismo de la ciudad y el poco realismo de esa distinción administrativa, que aún estaba vigente en 1887 (pero no en 1900). 
CUADRO 5

ACCESO A LA RED FERROVIARIA Y CRECIMIENTO URBANO

\begin{tabular}{|l|c|c|c|c|c|c|}
\hline \multirow{2}{*}{ Año } & \multicolumn{2}{|c|}{ Número de ciudades } & \multicolumn{3}{|c|}{ Crecimiento urbano } \\
\cline { 2 - 3 } \cline { 5 - 7 } & Sin acceso & Con acceso & Periodo & Sin acceso & Con acceso & Diferencia \\
\hline 1860 & 182 & 33 & & & & \\
\hline 1877 & 107 & 108 & $1860-1877$ & 0,48 & 0,81 & 0,33 \\
\hline 1887 & 72 & 143 & $1877-1887$ & 0,55 & 0,88 & 0,33 \\
\hline 1900 & 48 & 167 & $1887-1900$ & 0,29 & 0,52 & 0,23 \\
\hline 1910 & 45 & 170 & $1900-1910$ & 0,64 & 0,89 & 0,25 \\
\hline
\end{tabular}

Fuente: censos de población y García Raya (2006).

hacer comparables los incrementos de población independientemente del tamaño de las ciudades.

La variable «ferrocarril» ofrece muchas menos dificultades. Existe suficiente información para conocer la fecha de apertura de las estaciones ferroviarias en cada ciudad ${ }^{18}$. De hecho, es excesiva, pues las limitaciones de los censos de población sólo nos permiten discriminar dentro de los amplios periodos señalados por los censos de población. Al tratarse de una variable cualitativa, para cada uno de esos periodos determinaremos con un «1» si una ciudad estaba conectada a la red ferroviaria, y con un «0» si no lo estaba. Por supuesto, el hecho de que una ciudad tenga estación no significa que esté integrada en la red y, por tanto, pueda obtener todas las ventajas derivadas del acceso a un gran mercado. Lo consideraremos así cuando, a través del resto de la vía, sea posible acceder a Madrid, Barcelona, Valencia o Sevilla.

El número de localidades que en cada periodo contaron con un ferrocarril aparece recogido en el Cuadro 5. De las 215 localidades de la muestra, en 1877 la mitad ya tenían acceso; y sólo 45 seguían sin tenerlo en 1910. También puede observarse en las dos últimas columnas cómo el crecimiento demográfico de las ciudades sin tren fue significativamente más bajo que el de las que contaron con él, aunque esa diferencia parece haberse atenuado con el paso del tiempo. Esto constituye un indicio de los efectos positivos del ferrocarril en el desarrollo urbano. En el siguiente epígrafe trataremos de comprobarlo.

Varios de los modelos empleados también utilizan otras variables de control cuya función es eliminar el efecto que sobre el crecimiento urbano pudieron tener diversas circunstancias locales. Sobre la base de la bibliografía existente hemos identificado tres: la condición de capital de provincia, el acceso al mar y la existencia de una actividad industrial y minera relevante. Las dos primeras

${ }^{18}$ Véase García Raya (2006). 
no ofrecen dificultad para su determinación. Quizás sólo merezca la pena advertir que la condición de costa es estricta; es decir, se califica como tal aquella ciudad que tiene acceso al mar a través de un puerto propio o muy cercano, como Valencia y El Grao.

La condición industrial o minera encierra más problemas. En rigor, todas las localidades de la muestra tenían alguna actividad artesanal o industrial, por lo que cabría tentar una medición. Sin embargo, las dificultades documentales son, a nuestro juicio, insuperables. En la mayor parte de los casos se trataba de instalaciones antiguas y poco capitalizadas que subsistían al amparo de un nicho de mercado con escasa competencia. No pocas veces, esa actividad era complementaria de otra agrícola o comercial. No parece que tenga mucho sentido una medida de la industrialización que incluya todo tipo de actividades artesanales. Si lo que queremos es recoger el impacto de la industria sobre el crecimiento urbano sería necesario distinguir los talleres modernos, con una mayor productividad, salarios relativamente elevados y capacidad para atraer mano de obra, de los tradicionales, que en muchos casos fueron desapareciendo por la competencia que los otros ejercían. Esa información desagregada por localidades no existe. En los censos de 1860 y 1887, se clasifica a la población atendiendo a su ocupación principal, pero por partidos judiciales y provincias. En el de 1877, sólo aparecen datos provinciales. Y en los de 1900 y 1910, los de las provincias y sus capitales. Las ausencias y la falta de continuidad sugieren que, en realidad, la propia Administración no confiaba en sus datos, salvo donde disponía de mejores medios de control; es decir, en las capitales de provincia ${ }^{19}$.

Por estos motivos, hemos adoptado un criterio semejante al empleado en las otras variables de control; es decir, considerar a la minería e industria como una variable cualitativa e idiosincrática en la que sólo caben dos posibilidades: su existencia o inexistencia, lo que identificamos con un «1» $\mathrm{o}$ un «0», respectivamente. Determinar qué ciudades de la muestra eran mineras no es difícil; pero sí lo es determinar cuáles eran industriales. Nos hemos decidido por una muestra que, además de contar con industrias más o menos modernizadas, mantuvieron esa actividad a lo largo de todo el periodo de estudio. Para este propósito nos hemos servido, por un lado, de la bibliografía disponible, y en particular del Atlas de la Industrialización elaborado por Nadal, Benaul y Sudrià (2003); y, por otro lado, del censo de población de 1887 y sus datos por partidos judiciales. En resumen, 38 ciudades, 20 industriales y 18 mineras. $^{20}$

19 Véase Tirado, Pons y Paluzie (2006, pp. 50-52).

20 En concreto, hemos prestado especial atención al peso que en ese censo (vol. 2, pp. 472-491) tenía la población urbana masculina de entre 21 y 40 años dedicada «a la industria fabril y minera y a las derivadas de las mismas» en cada partido judicial, que incluye la ciudad analizada. Se han excluido algunas que vivieron procesos de desindustrialización, como Antequera, Requena y Marbella. En definitiva, las ciudades industriales serían Alcoy, Badalona, Barcelona, Béjar, Bilbao, Castro Urdiales, Elche, Gijón, Igualada, Manresa, Mataró, Reus, Sabadell, Sant Felíu de Guixols, 


\section{ANÁLISIS EMPÍRICO Y RESULTADOS}

Con objeto de cuantificar la influencia del ferrocarril sobre la población de cada municipio entre 1860 y 1910, comenzamos especificando un modelo de panel en diferencias en el que el crecimiento urbano $\left(p o b_{i t}\right)$, en porcentaje anual, es función del acceso a la vía férrea (tren ${ }_{i t}$ ), de otros factores explicativos $\left(\mathbf{X}_{i}\right)^{21}$ y de efectos fijos temporales (EFT). Éstos miden el resto de factores que influyen en el crecimiento urbano y que son constantes a todos los municipios pero que varían de un periodo a otro. Por construcción tienen media nula:

$$
\operatorname{pob}_{i t}=\beta_{0}+\beta_{1} \cdot \operatorname{tren}_{i t}+\mathbf{X}_{i}^{\prime} \boldsymbol{\delta}+E F T_{t}+\varepsilon_{i t},
$$

$\mathbf{X}_{i}$ es un vector que incorpora las variables de control, es decir, la capitalidad de provincia, su situación costera y el carácter minero o industrial de los municipios. Como hemos señalado en la sección anterior, la lógica económica e histórica sugiere que estas variables no ejercieron una influencia negativa sobre el crecimiento urbano $\left(\beta_{0}>0, \beta_{1}>0 \text { y } \boldsymbol{\delta}>\boldsymbol{0}\right)^{22}$. Al ser un modelo en diferencias, $\beta_{0}$ estima el crecimiento medio anual de las poblaciones sin acceso al ferrocarril y $\beta_{0}+\beta_{1}$, el crecimiento de los municipios que tuvieron acceso a él, descontando el efecto del resto de variables utilizadas.

La columna 1.1 del Cuadro 6 recoge la estimación en diferencias de panel de este modelo. Los resultados indican un crecimiento medio anual de los municipios sin acceso al tren del 0,32\%, mientras que los municipios con acceso añadían un $0,18 \%$ adicional. Es decir, estas poblaciones crecían un $58 \%$ más $\left[\left(\beta_{1} / \beta_{0}\right) \cdot 100\right]$ que las que no tuvieron acceso al ferrocarril. Las variables de control también aportan información útil. Así, las variables minería e industria y la capitalidad de provincia presentan porcentajes de crecimiento anual superiores a los del ferrocarril (0,77\% y 0,37\%, respectivamente). Evidentemente, las migraciones internas explican este fenómeno. Durante los años contemplados por este estudio, muchos campesinos emigraron hacia las capitales de provincia y las localidades industriales o mineras. La principal razón fue la disponibilidad de mejores servicios y la mejor calidad de vida en general; pero también la existencia de mayores oportunidades de trabajo, especialmente en el segundo tipo de ciudades ${ }^{23}$. La influencia de la costa sería

\footnotetext{
(F'note continued)

San Fernando, Terrassa, Valls, Vich, Vigo y Vilanova i la Geltrú. Las mineras serían Almadén, Almodóvar, Azuaga, Belmez, Berja, Cartagena, Cehegín, Cuevas, La Carolina, La Unión, Langreo, Linares, Mazarrón, Mieres, Minas de Ríotinto, Pozoblanco, Totana y Vera.

21 Para analizar posibles problemas de multicolinealidad, se han calculado dos estadísticos: el factor de inflación de varianza y la denominada condition number. Ninguno ofrece resultados preocupantes.

22 Lo que implica que los contrastes de significatividad de los estimadores serán de una sola cola $\left(\mathrm{H}_{0}: \beta_{i}=0\right.$ frente a $\left.\mathrm{H}_{1}: \beta_{i}>0\right)$.

${ }^{23}$ Véanse Silvestre (2005, pp. 246-250) y Rueda (2006, pp. 25-34).
} 
similar a la del ferrocarril, un crecimiento del $0,18 \%$ anual respecto de los municipios que no tenían esa condición.

Indudablemente, el hecho de que el crecimiento medio anual de las poblaciones con acceso al tren fuera mayor puede interpretarse de forma causal directa; pero el acceso al ferrocarril tampoco pudo ser independiente de la importancia de las ciudades, de su riqueza, su tamaño o su perspectiva de crecimiento. Fishlow (1965) lo planteó de la siguiente forma: ¿Tienen los ferrocarriles un impacto sustancial en el crecimiento o son consecuencia de él? Probablemente ambas afirmaciones son ciertas y el ferrocarril es consecuencia del crecimiento y a su vez causa de uno mayor (simultaneidad) ${ }^{24}$. También podría haber variables omitidas que se correlacionan con el crecimiento de la ciudad y el acceso al ferrocarril. Ambos problemas, causalidad inversa $\mathrm{y}$ variables omitidas, son potencialmente graves y pueden hacer que el estimador de la influencia del acceso a la vía férrea sobre el crecimiento urbano $\left(\beta_{1}\right.$ del modelo) sea sesgado e inconsistente. Para resolver ambos problemas recurrimos a dos enfoques diferentes. Para tratar la causalidad inversa estimaremos el modelo por variables instrumentales (VI) y mediante el uso de submuestras. El problema de variables omitidas lo trataremos estimando un modelo de panel en diferencias con efectos fijos temporales y transversales. Finalmente, para reforzar la conexión causal entre el acceso a la red ferroviaria y la urbanización, estimaremos un modelo de diferencias en diferencias donde la aceleración del crecimiento urbano es función del ferrocarril.

El tratamiento habitual de la endogeneidad consiste en estimar la ecuación por VI, lo que implica encontrar instrumentos válidos. La construcción de la vía férrea lleva tiempo, de modo que la decisión de unir dos ciudades necesariamente se tuvo que tomar a partir de información anterior a su efectiva realización. Por tanto, podemos considerar como instrumentos algunas de las variables retardadas del modelo, como el incremento de la población (lo que sería una variable aproximada del potencial crecimiento) o de sus habitantes (variable proxy de su importancia). Esto es una práctica habitual en Econometría. Nosotros hemos empleado como variables instrumentales el incremento de población anual en niveles, retardado un periodo ${ }^{25}$, pob (-1) del Cuadro 6, y también el acceso al tren retardado, tren (-1).

El procedimiento empleado en estos casos es el de mínimos cuadrados bietápicos. La estimación de la primera etapa para el acceso al tren se reproduce en la columna 1.2. De sus resultados se desprende que los dos instrumentos son significativos y, por consiguiente, adecuados para estimar el modelo por VI. La estimación por este método (segunda etapa) se muestra

${ }^{24}$ También es razonable suponer que la industria pueda plantear un problema de este tipo. Sin embargo, no creemos que esta dificultad pueda sesgar mucho los resultados, ya que sólo 20 ciudades de un total de 215 (el 9,3\%) tienen la condición de industriales. Como veremos en los diferentes modelos, su peso efectivo es aún inferior a ese $9 \%$.

${ }^{25}$ La utilización de la población en niveles retardada da resultados similares y por ello no la reproducimos en el Cuadro 2 . 


\section{CUADRO 6}

MODELOS EN DIFERENCIAS DE PANEL

\begin{tabular}{|c|c|c|c|c|c|c|}
\hline & \multirow{3}{*}{$\frac{\text { Original }}{1.1}$} & \multicolumn{2}{|c|}{ VI } & \multicolumn{2}{|c|}{ Submuestras } & \multirow{3}{*}{$\frac{\mathrm{EFT}+\mathrm{EFC}}{1.6}$} \\
\hline & & \multirow{2}{*}{$\begin{array}{c}1.2 \\
1^{\mathrm{a}} \text { etapa } \\
\text { Sin tren }\end{array}$} & \multirow{2}{*}{$\begin{array}{c}1.3 \\
2^{\mathrm{a}} \\
\text { Etapa }\end{array}$} & \multirow{2}{*}{$\begin{array}{c}1.4 \\
\begin{array}{c}\text { Sin } \\
\text { capitales }\end{array}\end{array}$} & \multirow{2}{*}{$\begin{array}{c}1.5 \\
\text { Sin } \\
1 / 4\end{array}$} & \\
\hline & & & & & & \\
\hline $\begin{array}{l}\text { Constant } \\
\mathrm{e}\end{array}$ & $\begin{array}{c}\mathbf{0 , 3 1 7} \\
(0,072) \\
{[0,000]}\end{array}$ & $\begin{array}{c}\mathbf{0 , 2 6 6} \\
(0,032) \\
{[0,000]}\end{array}$ & $\begin{array}{c}\mathbf{0 , 3 3 5} \\
(0,098) \\
{[0,000]}\end{array}$ & $\begin{array}{c}\mathbf{0 , 3 5 7} \\
(0,076) \\
{[0,000]}\end{array}$ & $\begin{array}{c}\mathbf{0 , 3 3 9} \\
(0,084) \\
{[0,000]}\end{array}$ & $\begin{array}{c}\mathbf{0 , 4 8 8} \\
(0,106) \\
{[0,000]}\end{array}$ \\
\hline Tren & $\begin{array}{c}\mathbf{0 , 1 8 5} \\
(0,088) \\
{[0,018]}\end{array}$ & & $\begin{array}{c}\mathbf{0 , 2 0 4} \\
(0,117) \\
{[0,041]}\end{array}$ & $\begin{array}{c}\mathbf{0 , 1 5 1} \\
(0,095) \\
{[0,056]}\end{array}$ & $\begin{array}{c}\mathbf{0 , 2 2 0} \\
(0,101) \\
{[0,015]}\end{array}$ & $\begin{array}{c}\mathbf{0 , 2 7 9} \\
(0,154) \\
{[0,035]}\end{array}$ \\
\hline Costa & $\begin{array}{c}\mathbf{0 , 1 8 3} \\
(0,125) \\
{[0,072]}\end{array}$ & $\begin{array}{l}\mathbf{- 0 , 0 1 9} \\
(0,030) \\
{[0,539]}\end{array}$ & $\begin{array}{c}\mathbf{0 , 1 5 2} \\
(0,140) \\
{[0,139]}\end{array}$ & $\begin{array}{c}\mathbf{0 , 1 3 1} \\
(0,151) \\
{[0,192]}\end{array}$ & $\begin{array}{c}\mathbf{0 , 3 5 8} \\
(0,180) \\
{[0,024]}\end{array}$ & \\
\hline Capital & $\begin{array}{c}\mathbf{0 , 3 6 6} \\
(0,092) \\
{[0,000]}\end{array}$ & $\begin{array}{c}\mathbf{0 , 0 8 4} \\
(0,023) \\
{[0,000]}\end{array}$ & $\begin{array}{c}\mathbf{0 , 3 5 3} \\
(0,108) \\
{[0,000]}\end{array}$ & & $\begin{array}{c}\mathbf{0 , 4 3 1} \\
(0,126) \\
{[0,000]}\end{array}$ & \\
\hline $\begin{array}{l}\text { Minería } \\
\text { o } \\
\text { industria }\end{array}$ & $\begin{array}{c}\mathbf{0 , 7 6 9} \\
(0,215) \\
{[0,000]}\end{array}$ & $\begin{array}{c}\mathbf{0 , 0 1 8} \\
(0,032) \\
{[0,573]}\end{array}$ & $\begin{array}{c}\mathbf{0 , 6 0 1} \\
(0,234) \\
{[0,006]}\end{array}$ & $\begin{array}{c}\mathbf{0 , 7 2 7} \\
(0,225) \\
{[0,000]}\end{array}$ & $\begin{array}{c}\mathbf{0 , 7 5 5} \\
(0,266) \\
{[0,002]}\end{array}$ & \\
\hline Tren (-1) & & $\begin{array}{c}\mathbf{0 , 7 1 8} \\
(0,031) \\
{[0,000]}\end{array}$ & & & & \\
\hline Pob $(-1)$ & & $\begin{array}{l}-\mathbf{0 , 0 1 3} \\
(0,007) \\
{[0,052]}\end{array}$ & & & & \\
\hline \multicolumn{7}{|l|}{ Tren $(+1)$} \\
\hline Tren/Cons. & 0,58 & & 0,61 & 0,42 & 0,65 & 0,57 \\
\hline EF, Temp. & Sí & Sí & Sí & Sí & Sí & Sí \\
\hline EF, Trans. & No & No & No & No & No & Sí \\
\hline Obs. & $\begin{array}{c}215 \\
(860)\end{array}$ & $\begin{array}{c}215 \\
(645)\end{array}$ & $\begin{array}{c}215 \\
(645)\end{array}$ & $\begin{array}{c}167 \\
(668)\end{array}$ & $\begin{array}{c}162 \\
(648)\end{array}$ & $\begin{array}{c}215 \\
(860)\end{array}$ \\
\hline $\mathrm{R}^{2}$ & 0,098 & 0,638 & 0,074 & 0,078 & 0,107 & 0,439 \\
\hline
\end{tabular}

Nota: se han incluido los errores estándar $\operatorname{robustos}^{26}$ de cada parámetro estimado entre paréntesis y su significatividad exacta ( $p$-valor) entre corchetes. En general, se utilizan contrastes de significatividad de una sola cola, excepto para el modelo 1.2, donde se emplea el contraste habitual de dos colas.

${ }^{26}$ Lo que significa que podemos realizar inferencia incluso en presencia de autocorrelación y heterocedasticidad. Véase Wooldridge (2002, pp. 148-53) y Arellano (1987) para un análisis de la utilización de errores estándar robustos en presencia de autocorrelación serial y heterocedasticidad 
en la columna 1.3. Las conclusiones son similares a las del modelo anterior, si bien ahora el crecimiento anual del grupo de control (ciudad sin acceso al tren, ni costa, ni capitalidad, ni carácter minero o industrial) habría aumentado ligeramente $(0,33 \%)$. Y también la influencia del ferrocarril $(0,20 \%)$. Por el contrario la influencia de la capitalidad de provincia y el carácter minero o industrial habrían disminuido $(0,35 \%$ y $0,60 \%)$. La costa no es significativa, ni siquiera al $10 \%$, de modo que sólo tiene utilidad como variable de control en este modelo. Las ciudades con acceso al tren crecerían un $61 \%$ más $\left[\left(\beta_{1} / \beta_{0}\right) \cdot 100\right]$ que las del grupo de control, porcentaje muy similar al estimado en el modelo 1.1.

Para resolver este problema de endogeneidad, Atack et al. (2010), Hornung (2012) y Banerjee, Duflo y Qian (2012) recurren, entre otras técnicas, al empleo de variables instrumentales (VI) construidas sobre la base de la línea recta trazada entre ciudades importantes; lo que resulta muy poco realista en el caso español porque la complicada orografía imponía trayectos sinuosos. Un modo alternativo sería estimar este modelo para distintas submuestras en las que se han suprimido las ciudades cuya interconexión habría sido el objetivo inicial del trazado ferroviario. Al actuar de este modo suponemos que las ciudades que quedan obtuvieron acceso al ferrocarril por azar. Si los resultados de los modelos de las submuestras son semejantes a los del modelo original 1.1, podemos colegir que, en efecto, no existe tal problema de endogeneidad. Este procedimiento también ha sido empleado por Hornung (2012). De esta forma, se elimina el sesgo, aunque al coste de reducir la muestra. La cuestión fundamental, entonces, es determinar cuáles eran esas ciudades importantes.

A pesar de que la legislación inicial, y en particular la ley de ferrocarriles de 1855 , tenía una voluntad ordenadora escasa o nula ${ }^{27}$, en la práctica, se fueron perfilando pronto las pautas de construcción de la red. Básicamente, ésta evolucionó alcanzando las principales ciudades españolas que en cada momento seguían sin estar conectadas ${ }^{28}$. Primero con Madrid y las mayores capitales, y luego con las siguientes en orden decreciente. De hecho, la conexión de todas las capitales de provincia fue un objetivo expresamente señalado en la segunda ley de ferrocarriles, de 1877; pero también se incluyó, y con carácter preferente, en los planes ferroviarios de 1867 y $1870^{29}$.

Esta lógica justifica la construcción de las diferentes submuestras. En primer lugar, la columna 1.4 reproduce la estimación del modelo en el caso de

\footnotetext{
(F'note continued)

en modelos de panel. Debemos tener en cuenta que, en general, los modelos de variables cualitativas con información transversal, como los utilizados en este trabajo presentan problemas de heterocedasticidad prácticamente en todos los casos.

27 Véase Wais (1974, p. 133).

28 Véase Capel (2007).

${ }^{29}$ Véase Mateo del Peral (1978, pp. 122-31).
} 
que eliminásemos las capitales de provincia. Según sus resultados, las ciudades del grupo de control habrían crecido, anualmente, un $0,36 \%$, mientras que la influencia del ferrocarril sería del $0,15 \%$. El porcentaje de crecimiento imputable a este factor sobre el grupo de control sería del $42 \%\left[\left(\beta_{1} / \beta_{0}\right) \cdot 100\right]$, lo que es un poco inferior al estimado en el modelo original 1.1. De modo alternativo, podemos considerar como ciudades importantes, simplemente, a las de mayor tamaño. La estimación de la columna 1.5 está construida con una muestra de la que se han detraído las ciudades que pertenecen al primer cuartil según su población. De nuevo, la influencia del ferrocarril es significativa a los niveles usuales. El crecimiento urbano atribuible al acceso a la red habría sido de un $0,22 \%$ anual, y el porcentaje de crecimiento imputable al ferrocarril sobre el grupo de control llegaría al $65 \%\left[\left(\beta_{1} / \beta_{0}\right) \cdot 100\right]$, que es ligeramente superior al obtenido en 1.1. Es interesante notar que en estos dos modelos el número de ciudades industriales eliminadas es proporcionalmente más elevado que el del conjunto de la muestra.

Como mencionamos antes, otra circunstancia que puede dar lugar a estimadores sesgados es el problema de las variables omitidas. Para tratar esta cuestión, podemos estimar el modelo 1 incluyendo efectos fijos de corte transversal (EFC). Éstos miden la influencia de factores idiosincráticos que inciden de forma diferente en el crecimiento (o decrecimiento) de la población de cada municipio, pero que no cambian con el tiempo. Como los EFT, tienen media nula. Las variables de control $\left(\mathbf{X}_{i}\right)$ que hemos venido manejando quedarían incluidas dentro de los EFC, que también recogerían cualquier otra variable con los requisitos anteriores.

Los resultados de la estimación de este modelo se presentan en el modelo 1.6. El grupo de control (las ciudades que no tuvieron acceso al tren una vez descontada la influencia del resto de factores considerados) creció un $0,49 \%$ anual mientras que la influencia del acceso al tren supone un $0,28 \%$ anual adicional. Respecto del grupo de control, la influencia del acceso a la red ferroviaria sería de un $57 \%\left[\left(\beta_{1} / \beta_{0}\right) \cdot 100\right]$, porcentaje similar a los obtenidos en las especificaciones $1.1,1.3$ y 1.5 .

En resumen, los resultados recogidos en el Cuadro 6 son una evidencia sólida del efecto causal del ferrocarril sobre el crecimiento de la población. Una vez descontado el efecto de las variables incluidas en $\mathbf{X}_{\mathbf{i}}$, las ciudades que dispusieron de la nueva tecnología experimentaron tasas de crecimiento superiores a las que no pudieron servirse de ella. Así, mientras que las ciudades sin acceso al ferrocarril presentaron porcentajes de crecimiento entre el $0,3 \%$ y el $0,5 \%$, las que dispusieron de la nueva tecnología añadieron en torno a un 0,2\% más. El crecimiento adicional parece mayor en las capitales, toda vez que el valor mínimo para el estimador de la variable tren, 0,151 , se registra en el modelo en el que éstas han sido excluidas. Ello podría estar indicando la existencia de algún tipo de efecto de interacción entre capitalidad y ferrocarril. Sea como fuere, el estimador que mide la influencia del ferrocarril siempre es positivo y estadísticamente significativo 
(al menos al 10\%), tanto en las estimaciones de las submuestras como en las que emplean variables instrumentales. El cociente «Tren/Constante» presenta un considerable grado de estabilidad, en torno a 0,6, indicando que el porcentaje de crecimiento de las ciudades que dispusieron de vía férrea fue un $60 \%$ superior al de aquellas que no contaron con ella.

Una aproximación alternativa para tratar de medir la causalidad y los efectos de la implantación del ferrocarril consiste en diseñar un experimento natural o cuasi experimento, e intentar medir el efecto causal mediante el estimador de diferencias en diferencias ${ }^{30}$. El experimento que proponemos consiste en comparar la aceleración del crecimiento urbano en los dos grupos, de tratamiento y de control, durante el periodo en el que se tuvo acceso a la nueva tecnología. Para ello especificamos un modelo en el que el incremento del porcentaje de crecimiento urbano $\left(\Delta p o b_{i t}\right)$ es función de $\Delta$ tren $_{i t}$. Tal como la hemos definido, esta última variable tomará el valor 1 sólo si se ha tenido acceso a la red en ese periodo. Es decir, un valor 0 no indica necesariamente que la ciudad no disponga de estación, sino sólo que no se ha puesto en funcionamiento en ese periodo. Además de las variables anteriores, incluimos, como antes, otros efectos explicativos $\mathbf{X}_{\mathbf{i}}$ y efectos fijos temporales (EFT). La ecuación que hay que estimar es:

$$
\Delta p o b_{i t}=\beta_{0}+\beta_{1} \cdot \Delta t r e n_{i t}+\mathbf{X}_{\mathbf{i}}^{\prime} \boldsymbol{\delta}+E F T_{t}+v_{i t},
$$

La variable explicada, al ser la diferencia de una tasa de variación, no se refiere al crecimiento sino a la aceleración (o desaceleración) del crecimiento urbano con respecto al periodo anterior. La variable $\Delta$ tren $_{i t}$ tiene, como hemos dicho, valor unitario en el periodo en que se tuvo acceso a la red ferroviaria y valor nulo en caso contrario. El resto de variables sólo tienen sentido como variables de control y no tienen interpretación causal. $\beta_{1}$ es el estimador de diferencias en diferencias y tiene la siguiente interpretación:

$$
\begin{aligned}
\beta_{1}= & \left(\text { crecimiento }^{\text {después }}-\text { crecimiento }^{\text {antes }}\right)^{\text {tratamiento }} \\
& -\left(\text { crecimiento }^{\text {después }}-\text { crecimiento }^{\text {antes }}\right)^{\text {control }}
\end{aligned}
$$

El Cuadro 7 reproduce dos estimaciones: la que se corresponde con la expresión (2) y la que sustituye $\mathbf{X}_{i}$ por efectos fijos de corte transversal.

Los dos modelos, columnas 2.1 y 2.2, presentan aceleraciones del crecimiento significativas al menos al 10\%, como consecuencia del acceso al tren $(0,39 \%$ y $0,46 \%$ anual, respectivamente). Por el contrario, el grupo de control o bien no tuvo aceleración (columna 2.2) o bien fue modesta $(0,12 \%$ anual, columna 2.1$)$. Como consecuencia del acceso al tren, la

\footnotetext{
${ }^{30}$ Véase Atack et al. (2010).
} 


\section{CUADRO 7}

MODELOS DE DIFERENCIAS EN DIFERENCIAS DE PANEL

\begin{tabular}{|l|c|c|}
\hline & 2.1 & 2.2 \\
\hline Constante & $\mathbf{0 , 1 1 5}$ & $\mathbf{0 , 0 1 8}$ \\
& $(0,041)$ & $(0,031)$ \\
{$[0,002]$} & {$[0,277]$} \\
\hline$\Delta$ Tren & $\mathbf{0 , 3 9 4}$ & $\mathbf{0 , 4 5 5}$ \\
& $(0,187)$ & $(0,320)$ \\
& {$[0,002]$} & {$[0,076]$} \\
\hline Costa & $\mathbf{- 0 , 0 7 0}$ & \\
\hline Capital & $\mathbf{- 0 , 1 6 2}$ & 25,28 \\
\hline Minería o industria & $\mathbf{- 0 , 2 5 5}$ & Sí \\
\hline$\Delta$ Tren/constante & 3,43 & Sí \\
\hline EF, Temporales & Sí & $215(645)$ \\
\hline EF, Transversales & No & 0,144 \\
\hline Observaciones & $215(645)$ & \\
\hline$R^{2}$ & 0,048 & \\
\hline
\end{tabular}

Nota: se presentan los errores estándar robustos entre paréntesis y la significatividad exacta ( $p$-valor) entre corchetes. Contrastes de significatividad de una sola cola.

aceleración se multiplicó por 3,43 respecto al grupo de control para el caso más modesto, y por 25,28 en el otro caso. Por tanto, parece que la influencia del acceso al ferrocarril tuvo un efecto causal claro sobre el crecimiento urbano, al menos en el periodo en que se tuvo acceso a él (este efecto además no se puede considerar de corto plazo, puesto que el periodo considerado es de, al menos, 10 años).

Este modelo es complementario a los estimados anteriormente. Nada impide que las tasas de crecimiento de las ciudades del grupo de control (con acceso anterior o sin acceso) crecieran de forma distinta a como lo hicieron las del grupo de tratamiento. Pero para que los resultados del Cuadro 6 fueran válidos sería necesario que no hubiera una aceleración de su crecimiento. Lo que constata el Cuadro 7 es que el crecimiento del grupo de tratamiento se aceleró, mientras que el del grupo de control no lo hizo (modelo 2.2) o lo hizo con moderación (modelo 2.1). Además, los modelos del Cuadro 7 prueban que en el periodo en el que tuvieron acceso al tren las ciudades aceleraron su crecimiento (efecto escalón). 


\section{CONCLUSIONES}

Este artículo trata de resolver, dentro del ámbito español, un problema observado por historiadores económicos de varios países: la debilidad de las pruebas sobre los efectos - positivos- de la construcción del ferrocarril en el crecimiento urbano. Teóricamente, es difícil negar la existencia de tales efectos; pero también es complicado observar un relación causa-efecto cuando los dos procesos están tan entrelazados con un tercero, la industrialización (y, probablemente, con otros). El hecho de que España fuera un país con una industria débil y muy localizada, pero con una extensa red ferroviaria, ayuda a plantear este problema de forma más nítida y, por tanto, a resolverlo mejor.

No obstante, el caso español presenta dificultades específicas. La principal es la escasez o ausencia de datos cuantitativos relevantes con los que contrastar esa hipótesis. Disponemos de censos de población para varios años, aunque a intervalos irregulares. Igualmente, conocemos las fechas de implantación del tren en cada una de las localidades del país. Pero no tenemos ninguna otra variable cuantitativa con la que explicar el crecimiento urbano. Teniendo en cuenta estos problemas hemos construido varios modelos que utilizan variables explicativas de naturaleza cualitativa.

Inicialmente, hemos estimado un modelo que relaciona el crecimiento urbano con el acceso al ferrocarril y tres variables de control y efectos fijos temporales. De dicho modelo se desprende una relación directa y positiva con un parámetro $\beta_{1}=0,185$. Es decir, la población de las ciudades con acceso a la red ferroviaria habría crecido un $0,185 \%$ anual adicional al de las ciudades que no contaron con esa tecnología, cuyo crecimiento total fue del 0,317\% anual.

Este modelo puede encerrar un hipotético problema de endogeneidad, para cuyo tratamiento hemos seguido una doble vía. En primer lugar, hemos estimado un modelo por VI (1.2 y 1.3) del que se desprende un coeficiente similar $(0,204 \%)$. También hemos utilizado submuestras en las que se han eliminado distintos grupos de ciudades (modelos 1.4 y 1.5), obteniendo estimadores igualmente próximos a los del modelo original. En ningún caso se puede rechazar la hipótesis $\mathrm{H}_{0}: \beta_{1}=0,185$. Todo ello sugiere que el sesgo, de existir, no debió de ser importante, y que el efecto del ferrocarril sobre la población puede cifrarse entre un $0,15 \%$ y un $0,28 \%$ adicional. Esto quiere decir que, con respecto al crecimiento de las ciudades sin ferrocarril, las que sí estuvieron conectadas tuvieron un crecimiento de alrededor de un $60 \%$ superior, cifra que muestra una notable estabilidad. Así pues, creemos que el modelo original mide de forma razonable el efecto causal del ferrocarril sobre el incremento de la población y que éste puede cifrarse aproximadamente en un $0,185 \%$ anual. La posible endogeneidad de la industria, de existir, no ha podido generar graves distorsiones, porque son muy pocas las ciudades que poseen esa condición.

Adicionalmente, hemos estimado dos modelos de diferencias en diferencias con objeto de medir el efecto que disponer de esta tecnología tenía 
sobre la aceleración del crecimiento en el periodo en el que el ferrocarril llegaba a cada ciudad. Los resultados muestran una fuerte aceleración del crecimiento anual, entre el 0,39 y el $0,45 \%$ anual.

En comparación con los valores obtenidos en otros trabajos, estas cifras pueden considerarse moderadas. Por ejemplo, el incremento que supuso la construcción del ferrocarril en el crecimiento de las ciudades prusianas entre 1849 y 1871 , según Hornung (2012), fue cinco veces mayor (0,9\% anual) del que hemos encontrado en España. De modo coherente, hemos visto que el intenso crecimiento de la red ferroviaria entre 1855 y 1873, así como el mantenimiento de la inversión hasta fin de siglo, vino acompañado de un crecimiento urbano semejante o algo inferior al del resto de Europa. En fin, la construcción del ferrocarril habría tenido efectos notables sobre la urbanización y la economía española, pero menos intensos que en otros países europeos. De esto cabría deducir que las estimaciones más modestas sobre el ahorro social también son las más verosímiles.

\section{BIBLIOGRAFÍA}

Akgüngör, S.; Aldemir, C.; Kustepeli, Y.; Gülcan, Y. y Tecim, V. (2011): «The Effect of Railway Expansion on Population in Turkey, 1856-2000». The Journal of Interdisciplinary History XLII (I) 6, pp. 135-157.

ANES, R. (1978): «Relaciones entre el ferrocarril y la economía», en M. Artola (dir.), Los ferrocarriles en España, 1844-1943. Madrid: Banco de España, vol. 2, pp. 355-512.

Arellano, M. (1987): «Computing Robust Standard Errors for Within-groups Estimators». Oxford Bulletin of Economics and Statistics 49, pp. 431-434.

Atack, J.; Bateman, F.; Haines, M. y Margo, R. A. (2010): «Did Railroads Induce or Follow Economic Growth? Urbanization and Population Growth in the American Midwest, 1850-1860». Social Science History 34, pp. 171-197.

Bairoch, P. (1988): Cities and economic development. Londres: Mansell.

Bairoch, P. y Goertz, G. (1986): «Factors of Urbanisation in the Nineteenth Century Developed Countries: A Descriptive and Econometric Analysis». Urban Studies 23, pp. 285-305.

Banerjee, A., Duflo, E. y Qian, N. (2012). «On the Road: Access to Transportation Infrastructure and Economic Growth in China». NBER Working Papers 17.897.

Bardet, J.-P. y Dupâouier, J. (dirs.) (1998): Historia de las poblaciones de Europa, vol. II: La revolución demográfica. Madrid: Síntesis.

Barouín, R. (1997): «Transporte y precio del trigo en el siglo XIX». Revista de Historia Económica 15 (1), pp. 17-48.

BARQuín, R. (1999): «El ahorro social: una perspectiva dinámica», en M. Muñoz, J. Sanz, y J. Vidal (eds.), Siglo y medio del ferrocarril en España, 1848-1998. Madrid: Fundación de los Ferrocarriles, pp. 337-354. 
CAmpo, S. Del (1972): Análisis de la población de España. Barcelona: Ariel.

CAPEL, H. (2007): "Ferrocarril, territorio y ciudades». Revista Bibliográfica de Geografía y Ciencias Sociales XII (717). Serie documental de Geo Crítica.

CAPEL, H. (2011): Los ferrocarriles en la ciudad. Redes técnicas y configuración del espacio urbano. Madrid: Fundación de los Ferrocarriles Españoles.

FIsHlow, A. (1965): American Railroads and the Transformation of the Antebellum Economy. Cambridge: Harvard University Press.

García RAYA, J. (2006): "Cronología básica del ferrocarril español de vía ancha». IV Congreso de Historia Ferroviaria. Málaga: Junta de Andalucía, Consejería de Obras Públicas y Transportes.

Gómez Mendoza, A. (1982): Ferrocarril y cambio económico en España 1855-1913. Madrid: Alianza.

Gómez Mendoza, A. y LunA, G. (1986): «El desarrollo urbano en España, 18601930». Boletín de la Asociación de Demografía Histórica 4 (2), pp. 3-22.

Gregory, I. N. y Martí-Henneberg, J. (2010): «The Railways, Urbanization, and Local Demography in England and Wales, 1825-1911». Social Science History 34/2, pp. 199-228.

Herranz, A. (2004): La dotación de infraestructuras en España (1844-1935). Madrid: Banco de España.

Herranz, A. (2008): Infraestructuras y crecimiento económico en España (1850-1935). Madrid: Fundación de los Ferrocarriles Españoles.

Herranz, A. (2012): Infraestructuras y crecimiento económico en España, 1850-1935. Madrid: Fundación de los Ferrocarriles Españoles.

Hirschman, A. O. (1958): The Strategy of Economic Development. New Haven: Yale University Press.

Hornung, E. (2012): «Railroads and Micro-regional Growth in Prussia». Ifo Working Paper 127.

Huisg, A., Koopmans, C. y Rietveld, P. (2010): «An Accessibility Approach to Railways and Municipal Population Growth, 1840-1930». 12 $2^{\text {th }}$ World Conference on Transport Research. Lisboa. En 〈http://intranet.imet.gr/ Portals/0/UsefulDocuments/documents/01884.pdf $\rangle$.

Kotavaara, O.; Antikainen, H. y Rusanen, J. (2011): «Urbanization and Transportation in Finland». The Journal of Interdisciplinary History XLII (I) 6, pp. 89-109.

Krugman, P. (1991): Geography and Trade. Cambridge, Mass: MIT Press.

LunA, G. (1988): «La población urbana en España, 1860-1930». Revista de Demografía Histórica 6 (1), pp. 25-68.

Mateo del Peral, D. (1978): «Los orígenes de la política ferroviaria en España (1844-1877)», en M. Artola (dir.), Los ferrocarriles en España, vol. 1. Madrid: Banco de España, pp. 31-159.

Matilla, M.; PÉRez, P. y SAnz, B. (2009): «La integración del mercado español a finales del siglo XIX: Los precios del trigo entre 1891 y 1905». Revista de Historia Económica 27, pp. 321-349. 
Mojica, L. y Martí-Henneberg, J. (2011): «Railways and population distribution: France, Spain and Portugal, 1870-2000». The Journal of Interdisciplinary History XLII (I) 6, pp. 15-28.

Morillas-Torné, M.; Franch, X.; Martí-Henneberg, J. y García, A. (2012): «Transformación urbana y desarrollo del ferrocarril en España, 1850-2000», 32 ${ }^{\text {nd }}$ International Geographical Congress. Cologne.

Nadal, J.; Benaul, J.-M. y Sudrià, C. (2003): Atlas de la industrialización de España. Barcelona: Crítica.

Pons, J.; Paluzie, E.; Silvestre, J. y Tirado, D. A. (2007): «Testing the New Economic Geography: Migrations and industrial agglomerations in Spain». Journal of Regional Science 7 (2), pp. 289-313.

Pons, J.; Paluzie, E.; Silvestre, J. y Tirado, D. A. (2009): «Migrants and market potential in Spain over the twentieth century: a test of the new economic geography». Spanish Economic Review 11, pp. 243-265.

PANTA, L. DEL (1989): «Population growth, urbanization and regional differentials in Italy during the Nineteenth Century (1796-1914)», en R. Lawton y R. Lee (eds.), Urban population development in Western Europe from the Late-Eighteenth to the early-twentieth century. Liverpool: Liverpool University Press, pp. 258-269.

Pérez MoredA, V. (1985): «La modernización demográfica, 1800-1930: sus limitaciones y cronología», en N. Sánchez-Albornoz (comp.), La modernización económica de España, 1830-1930. Madrid: Alianza, pp. 25-62.

Poussou, J. P. (1989): «The population increase of French towns between 1750 and 1914, and its demographic consequences», en R. Lawton y R. Lee (eds.), Urban population development in Western Europe from the Late-Eighteenth to the early-twentieth century. Liverpool: Liverpool University Press, pp. 68-92.

ReHer, D.-S. (1986): «Desarrollo urbano y evolución de la población: España 1787-1930». Revista de Historia Económica IV (1), pp. 39-66.

Reher, D.-S.; Nogueras, B. y Pombo, M. N. (eds.) (1993): España a la luz del Censo de 1887. Madrid: Instituto Nacional de Estadística.

Rietveld, P. y BRuinsma, F. R. (1998): Is Transport Infrastructure Effective? Berlín: Springer.

Rodríguez Osuna, J. (1985): Población y territorio en España. Siglos XIX y XX. Madrid: Espasa-Calpe.

Rosés, J.; Martínez-galarraga, J. y Tirado, D. A. (2010): «The upswing of regional income inequality in Spain (1860-1930)». Explorations in Economic History 47, pp. 244-257.

RuedA, G. (2006): España 1790-1900. Sociedad y condiciones económicas. Madrid, Istmo.

Schumpeter, J. A. (1939): Business Cycles: a theoretical, historical and statistical analysis of the capitalist process. Nueva York: McGraw-Hill.

Silvestre, J. (2005): «Internal migrations in Spain, 1877-1930». European Review of Economic History 9, pp. 233-265. 
Tirado, D. A.; Pons, J. y Paluzie, E. (2006): «Los cambios en la localización de la actividad industrial en España, 1850-1936. Un análisis desde la Nueva Geografía Económica». Revista de Historia Industrial 31 (XV) 2, pp. 41-62.

VALERO, A. (1989): «El sistema urbano español en la segunda mitad del siglo XIX». Boletín de la Asociación de Demografía Histórica 7 (VII) 1, pp. 7-29.

WaIs, F. (1974): Historia de los ferrocarriles españoles. Madrid: Editora Nacional.

Weber, A. F. (1899): The Growth of Cities in the Nineteenth Century: a study in statistics. Nueva York: Grennwood Press.

Wooldridge, J. M. (2002): Econometric Analysis of Cross Section and Panel Data. Cambridge: The MIT Press. 